\title{
DESARROLLO DE UN MODELO DE FLUJO VERTICAL EN TUBERÍAS PARA CRUDOS EXTRAPESADOS CONSIDERANDO EL FÉNOMENO DE FOAMY OIL
}

Carlos Andres Díaz Prada ${ }^{1}$, Nicolás Santos Santos ${ }^{2 *}$, Anibal Ordoñez Rodríguez ${ }^{3}$

*A quien debe dirigirse la correspondencia

\begin{abstract}
RESUMEN
Los fluidos tipo "Foamy Oil" tienen una gran particularidad en la dinámica de fases ante la variación de la presión y temperatura en el sistema. El presente artículo plantea una aproximación a partir de dos fenómenos: Nucleación y Coalescencia. El primero permite establecer la geometría que regula la dinámica de las fases y el segundo las condiciones a partir de las cuales la fase gaseosa empieza a fluir como fase independiente, esto es, las condiciones a partir de las cuales la geometría esférica de las burbujas de gas cambia a una geometría regulada por la burbuja de Taylor. El punto de encuentro de los dos se define a partir del modelo tipo "volúmenes igualados", por medio del cual se establece el diferencial de presión en términos de la reología del fluido, la cuales se puede expresar de acuerdo al modelo de ley de potencia para fluidos no-newtonianos. La evaluación del fenómeno se realiza en términos probabilísticos para asegurar que la sensibilidad de las variables establezcan los rangos de aplicabilidad y consistencia de los resultados.
\end{abstract}

Palabras clave: Foamy Oil, Ley de Potencia, Propiedades PVT, Nucleación, Coalescencia.

\section{VERTICAL FLOW MODEL DEVELOPMENT FOR EXTRAHEAVY OIL CONSIDERING FOAMY OIL PHENOMENON}

\begin{abstract}
Fluids that fall under the heading "Oil Foamy" represents a particularity in terms of characterizing properties as flow dynamics which can be represented from the dynamics of the phases before the pressure variation and system temperature. This paper examines an approach take into account two phenomena: nucleation and coalescence. The first one set the geometry, which defines phases dynamics and the second the conditions from which the gas phase begins to flow separately. That is, the conditions from which the spherical geometry, defined for gas bubbles modeling, change to a geometry regulated by the Taylor bubble, so that, the evaluated phenomena set the initial and boundary conditions for the model applicability.
\end{abstract}

Keywords: Foamy Oil, Power Law, PVT properties, Nucleation, Coalescence.

1. Magister en Ingeniería de Hidrocarburos. Instituto Colombiano del Petróleo. ICP. ECOPETROL. S.A. Piedecuesta Colombia.

2. Magister en Ingeniería de Hidrocarburos. Grupo de Modelamiento de Procesos de Hidrocarburos. Universidad Industrial de Santander, UIS. Bucaramanga. Colombia. E-mail: nicolas@uis.edu.co

3. Magister en Ingeniería de Hidrocarburos. Instituto Colombiano del Petróleo. ICP. ECOPETROL. S.A. Piedecuesta Colombia. E-mail: anibal.ordonez@ecopetrol.com.co. 


\section{INTRODUCCIÓN}

El crudo denominado Foamy Oil puede definirse como una fase continua líquida con alto contenido de gas que permanece disuelto durante condiciones en las que un gas asociado a un líquido convencional fluiría como fase independiente. El análisis de este fenómeno en la industria ha tenido un desarrollo notable referido a los crudos pesados, debido a que algunos yacimientos productores de este tipo de hidrocarburos, bajo un mecanismo de gas en solución, han tenido un factor de recobro mayor al que pudiera predecir cualquier modelo aplicable a los crudos convencionales. Por este motivo, al realizar un análisis de los valores de la presión en los distintos nodos del sistema de producción, se puede establecer que mientras la presión del yacimiento permanezca por encima de la presión de burbuja, el fenómeno de la solubilidad del gas cobra mayor preponderancia entre la cara de la formación y la cabeza de pozo.

El comportamiento del flujo de este fluido debe analizarse primero teniendo en cuenta sus propiedades, especialmente la solubilidad de gas que redefine el valor de presión de burbuja obtenido de manera convencional, por cuanto esta establece el umbral a partir del cual el gas se distribuye en esta unidad del sistema de producción. Los límites de este comportamiento se analizan en función de la velocidad del gas en la fase líquida, para lo cual se propone una función de distribución de probabilidad de frecuencia del tamaño de la burbuja en el sistema a un momento dado y el límite, que marca la separación de las fases, por medio del balance de fuerzas que se presenta sobre una burbuja de gas.

La función obtenida mediante este balance permite modificar la solubilidad del gas en el líquido, con lo cual se define un contenido de gas a unas condiciones del sistema a partir del valor más probable de separación de un diámetro de burbuja. El valor de la presión de burbuja se determina a partir de la modificación de las constantes de equilibrio entre las fases, con lo cual se establece que el cambio de las propiedades del fluido define su variación ante un diferencial de presión en el sistema. El balance que permite estimar el diferencial de presión del sistema se basa en la relación de Expansión Volumétrica, con lo cual, se incluye el efecto de la velocidad del gas en la unidad de análisis.

La correlación que resulta del desarrollo de este balance se compara con datos experimentales de un crudo extrapesado de un campo colombiano y se realiza un análisis estadístico de los datos obtenidos.

\section{ANÁLISIS}

El efecto de los parámetros que definen la geometría de flujo y las condiciones de operación del sistema, se determina mediante la profundidad a partir de la cual las burbujas se empiezan a separar del sistema, distancia a partir de la que coalescen para formar burbujas de mayor tamaño antes de formar el tamaño que se considera como límite del patrón que permite caracterizar el fluido como tipo Foamy Oil (Abou-el-hassan, 1983), (Akagawa \& Sakaguchi, 1966).

Para un fluido de las propiedades de un crudo extrapesado de un campo colombiano, que tiene una gravedad promedio de $8.5^{\circ} \mathrm{API}$, y un gas con una gravedad especifica de 0.8 , se tienen los siguientes valores:

Tabla 1. Propiedades del crudo extrapesado de un campo colombiano usado para la evaluación.

\begin{tabular}{|ccc|}
\hline Propiedad & Crudo & Gas Formación \\
\hline T $\left({ }^{\circ} \mathbf{F}\right)$ & 60 & 60 \\
\hline P (PSIA) & 14.7 & 14.7 \\
\hline SG & 1.023 & 0.8 \\
\hline MW & 416.3 & 22.88 \\
\hline Densidad Másica (lb/ft3) & 63.84 & $6.053 \mathrm{E}-02$ \\
\hline Densidad Molar (lb mol/ft3) & 0.1534 & $2.646 \mathrm{E}-03$ \\
\hline Tensión Superficial (dina/cm) & 35.22 & 24.14 \\
\hline
\end{tabular}

Considerando además las siguientes propiedades mecánicas de operación de los pozos como valores típicos:

Tabla 2. Propiedades mecánicas de pozos productores de crudo de la formación productora del crudo extrapesado.

\begin{tabular}{|cc|}
\hline Propiedad & Valor \\
PMP (ft) & 8000 \\
\hline Profundidad Bomba (ft) & 5000 \\
\hline THP (PSI) & $100-300$ \\
\hline Qo (BPD) & $250-1000$ \\
\hline BSW (\%) & $0.8-20 \%$ \\
\hline GOR producción (SCF/STB) & $5-250$ \\
\hline Tfondo $\left({ }^{\circ} \mathbf{F}\right)$ & 185 \\
\hline
\end{tabular}

Fenómeno de nucleación. La nucleación se ha analizado a partir de la dinámica de las burbujas en la fase líquida como el conjunto de condiciones a partir de las cuales estas coalescen en función de la movilidad que alcanzan en proporción al tamaño que logran en el medio de flujo. Normalmente, las variables que condicionan esta unión son la viscosidad del líquido y la tensión interfacial (Blauer \& Holcomb, 1975), (Blauer, Mitchell \& Kohlhaas, 1974). 
Al definir las variables operativas que pueden afectar el fenómeno de nucleación a partir de funciones de probabilidad, se tiene una cobertura más amplia de la profundidad a la cual se presentará una separación de la burbuja de tamaño promedio (Bonilla \& Shah, 2000).

Para el caudal de crudo se selecciona una distribución de tipo BetaPert (ANSYS ${ }^{\circledR}$ CFX-Solver.), en la que se incluye una mayor densidad entre los valores de 250 BPD y 600 BPD, pero alcanzando el límite superior definido en la tabla anterior y un límite inferior de hasta $100 \mathrm{BPD}$, tal y como se muestra en la siguiente figura:

(Se considera un gradiente de temperatura de $1^{\circ} \mathrm{F} / 100 \mathrm{ft}$.)

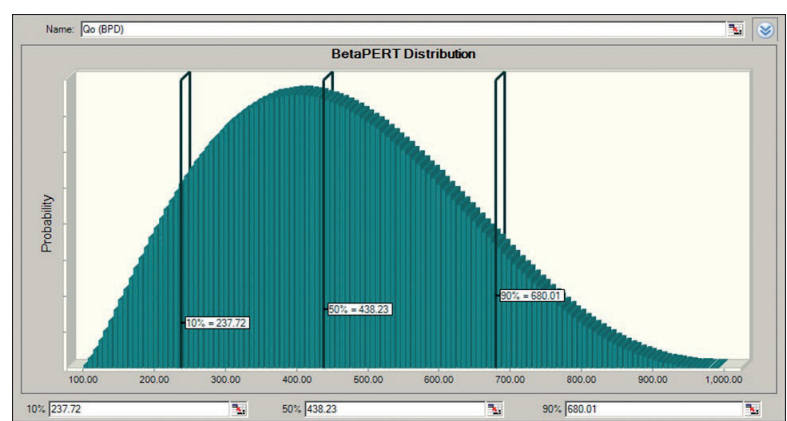

Figura 1. Distribución de valores de caudal de producción

Fuente: Autor

Los diámetros de la tubería de producción en el campo varían entre $95 / 8$ ", que se ubica en las secciones inferiores, y 5 1/2 “, que se ubica aguas debajo de la bomba de subsuelo. Se define una distribución triangular que contenga estos valores, pero que alcance valores de tubería de $27 / 8$ ", para corroborar el funcionamiento de la burbuja de Taylor (Bird, et al) como valor límite de la función de distribución en geometrías pequeñas. Se asume además una distribución continua debido a que el diámetro interno de la tubería puede variar a lo largo de la sarta de producción debido a depositación de orgánicos (Barnes et al).

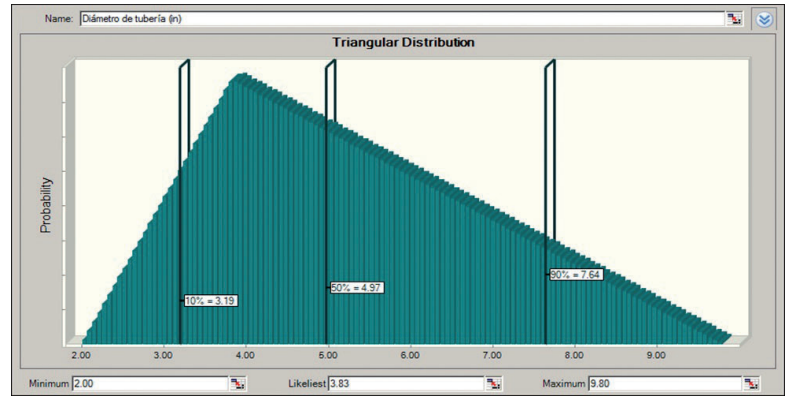

Figura 2. Distribución de valores de diámetros de tubería de producción. Fuente: Autor
La variación de la presión en cabeza de pozo se asume de tipo BetaPert (ANSYS ${ }^{\circledR}$ CFX-Solver.), condensando la mayor densidad de probabilidad en un valor cercano a los 200 PSI. Debido a la variación de la distancia de los pozos a la estación de tratamiento, se establece una función continua para dar cobertura a cualquier valor posible.

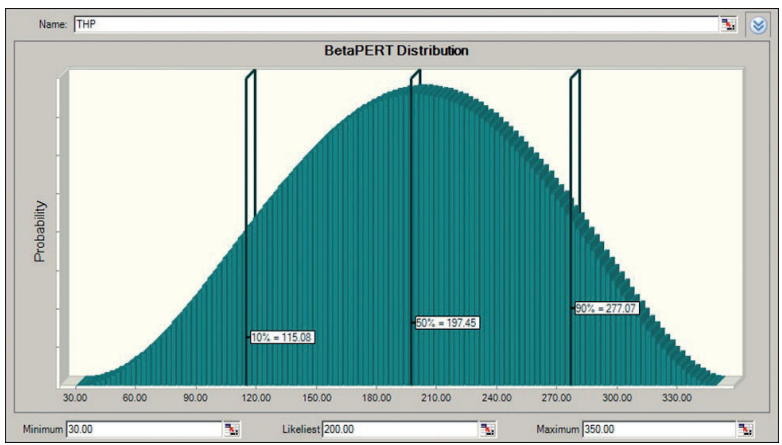

Figura 3. Distribución de valores de presión en cabeza de pozo Fuente: Autor

Finalmente, se asume una distribución de tipo Weibull (ANSYS ${ }^{\circledR}$ CFX-Solver.), para describir el comportamiento del tamaño de burbuja promedio del sistema, lo cual corresponde a lo definido en la sección de análisis del fenómeno de la nucleación.

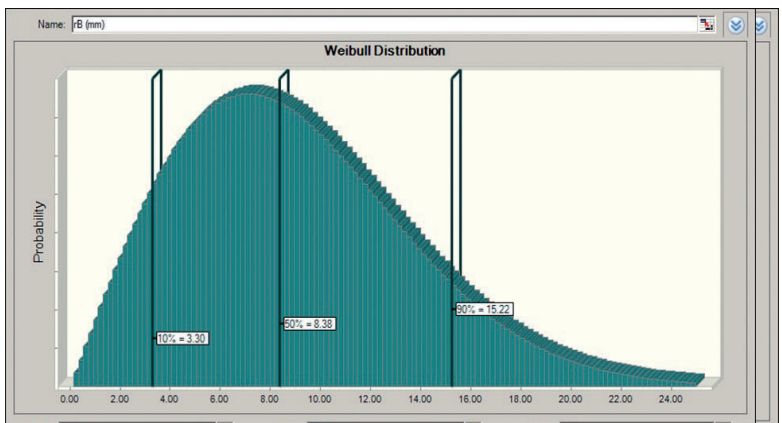

Figura 4. Distribución de valores de tamaños de burbuja

Fuente: Autor

Estas funciones definen la distribución de probabilidad de frecuencia de la profundidad a la cual se separa el gas del crudo, valor que define tanto la función de solubilidad del gas como la cantidad de gas presente como fase libre a ciertas condiciones del sistema (Calvert, J. R. and K. Nezhati). La función propuesta tiene el siguiente comportamiento:

Por su parte, las correlaciones restantes tienen el comportamiento mostrado en la figura 6 .

Se asume la distribución determinada a partir de la función que resulta de la presente investigación. 


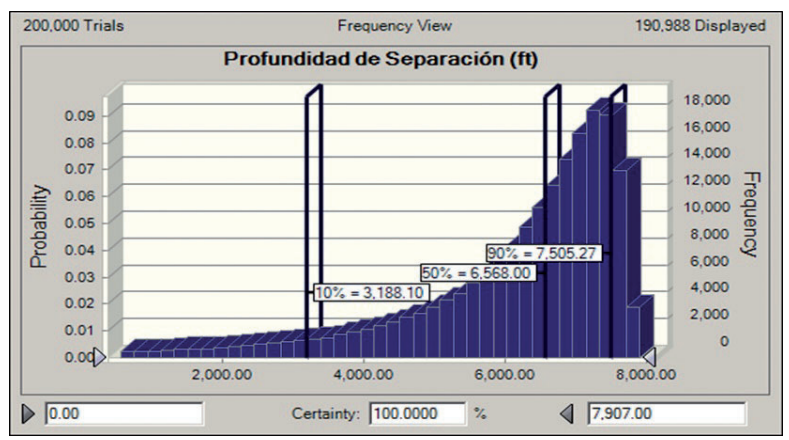

Figura 5. Distribución de valores de profundidad de separación para la función propuesta

Fuente: Autor

Los valores de control del gradiente de presión calculado por el modelo presentado en la presente investigación son los datos de registros PLT tomados en diferentes pozos del campo productor de crudo extrapesado utilizado para la evaluación, los cuales se muestran en la figura 7.

De este modo, la unidad de análisis se delimita por la región comprendida entre la profundidad promedio de la formación productora de crudo extrapesado y la profundidad de la entrada a la bomba. Pero, la comparación debe incluir además los valores definidos por las correlaciones más utilizadas para el modelamiento de flujo vertical (Beggs and Brill, Duns and Ros y Hagedorn and Brown), por un modelo mecanístico comúnmente usado (Ansari) y por un modelo mecanístico propuesto por la universidad de Tulsa (TUFFP) que fundamenta su desarrollo en el régimen de tipo slug y los límites que marca la distribución de las velocidades de las fases respecto a otros regímenes de flujo (David \& Marsden, 1969), (Gaddis \& Vogelpohl, 1986).
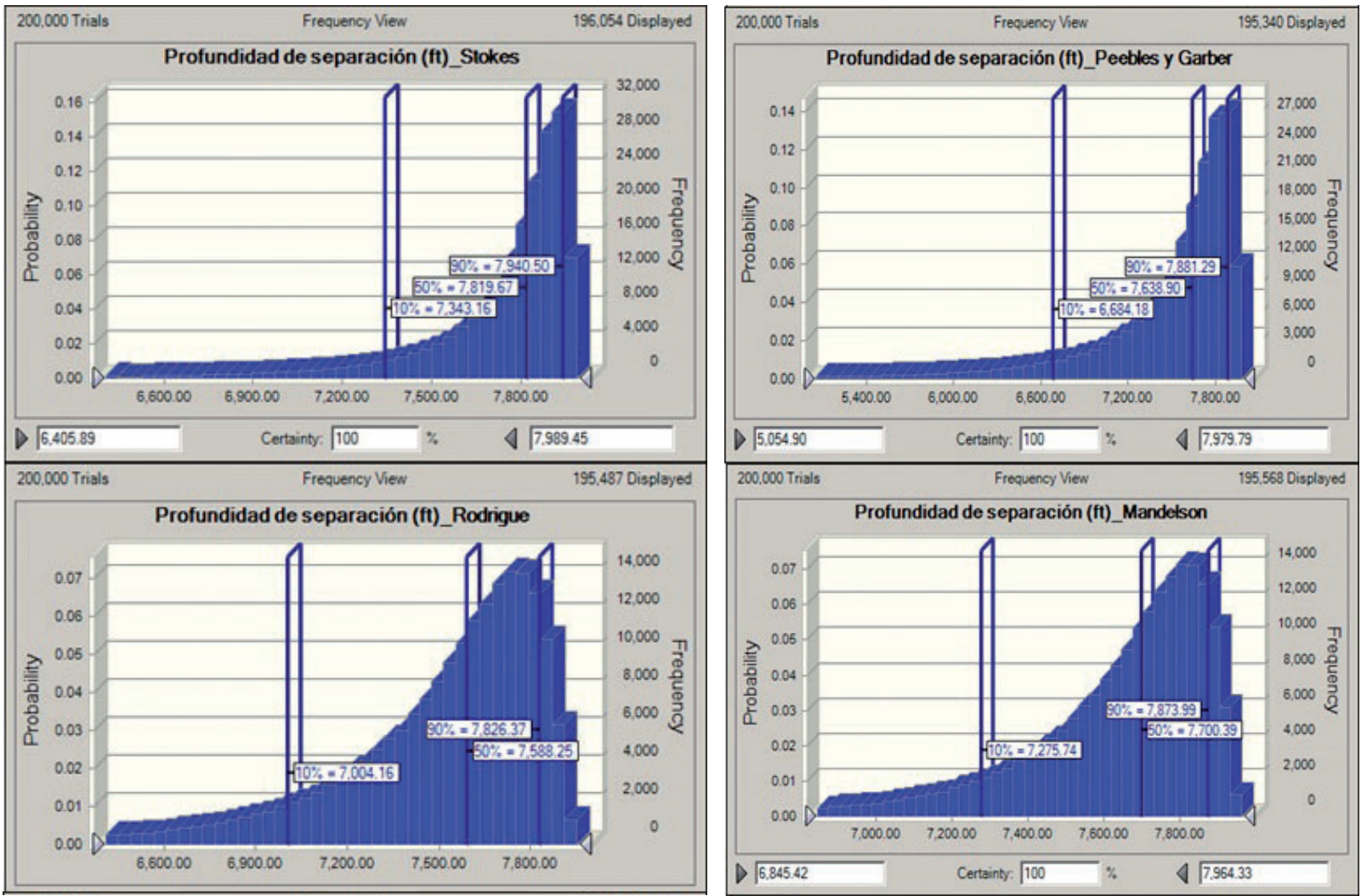

Figura 6. Distribución de valores de profundidad de separación en función de otras funciones de velocidad de gas en el sistema Fuente: Autor 


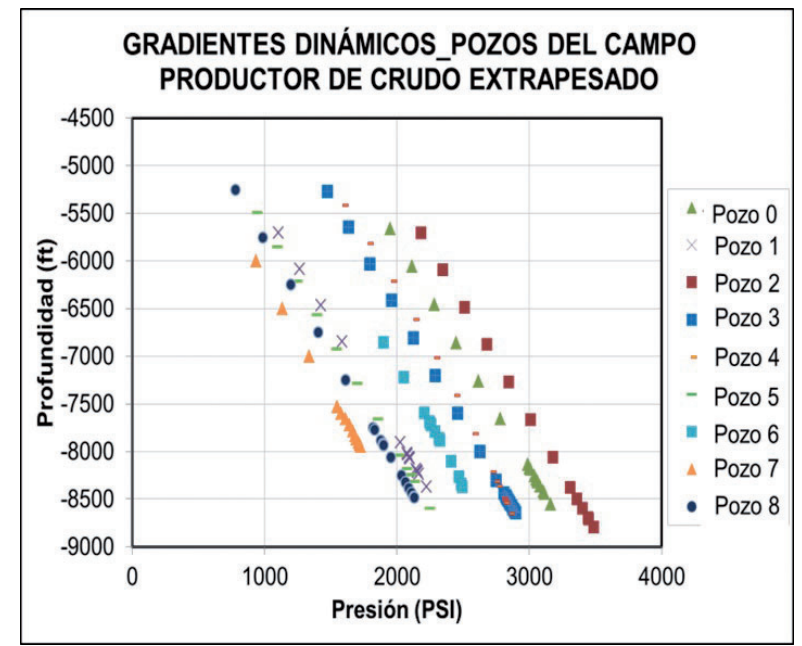

Figura 7. Gradientes dinámicos de pozos productores de crudo extrapesado de un campo colombiano

Fuente: Ecopetrol S.A.

Calculo del gradiente de presión, El cálculo del gradiente de presión mediante estas correlaciones tiene dos comportamientos muy marcados: presentan una diferencia que se torna cada vez mayor ante el ascenso del fluido o se mantienen invariables en todo el sistema (Gardiner, Dlugogorski \& Jameson, 1999), (Harris, P.C. and Heath, 1996). Las condiciones de los pozos son las siguientes:

Tabla 3. Propiedades de los pozos productores de crudo extrapesado evaluado.

\begin{tabular}{|c|c|c|c|c|c|c|c|c|c|}
\hline W & Qo & BSW & GOR & API & m1@150F & $\mathbf{m} 2 @ 180^{\circ} \mathbf{F}$ & Pws & Tyto & OD \\
\hline $\begin{array}{c}\text { POZO } \\
1\end{array}$ & 92 & 2 & 60 & 8 & 8488 & 2509 & 2698 & 180 & \\
\hline $\begin{array}{c}\text { Pozo } \\
2\end{array}$ & 678 & 10 & 50 & 10 & 4109 & 1177 & 2622 & 180 & \\
\hline $\begin{array}{c}\text { Pozo } \\
3\end{array}$ & 268 & 8 & 70 & 8 & 8488 & 2509 & 3380 & 180 & \\
\hline $\begin{array}{c}\text { Pozo } \\
4\end{array}$ & 340 & 12 & 110 & 8 & 8488 & 2509 & 3172 & 180 & \\
\hline $\begin{array}{c}\text { Pozo } \\
5\end{array}$ & 1897 & 60 & 147 & 8 & 4870 & 1799 & 2306 & 180 & $\begin{array}{c}9 \\
5 / 8\end{array}$ \\
\hline $\begin{array}{c}\text { Pozo } \\
6\end{array}$ & 64 & 22 & 80 & 9 & 6061 & 1954 & 2505 & 180 & \\
\hline $\begin{array}{c}\text { Pozo } \\
7\end{array}$ & 746 & 38 & 224 & 9 & 6789 & 1730 & 1764 & 180 & \\
\hline $\begin{array}{c}\text { Pozo } \\
8\end{array}$ & 1298 & 82 & 147 & 8 & 4870 & 1799 & 2015 & 180 & \\
\hline $\begin{array}{c}\text { pozo } \\
0\end{array}$ & 390 & 4 & 60 & 10 & 5700 & 1740 & 3065 & 180 & \\
\hline
\end{tabular}

De este modo, se puede integrar el resultado del modelo propuesto con los resultados obtenidos mediante estas correlaciones y el valor de los PLT del campo. Los resultados son los siguientes: 


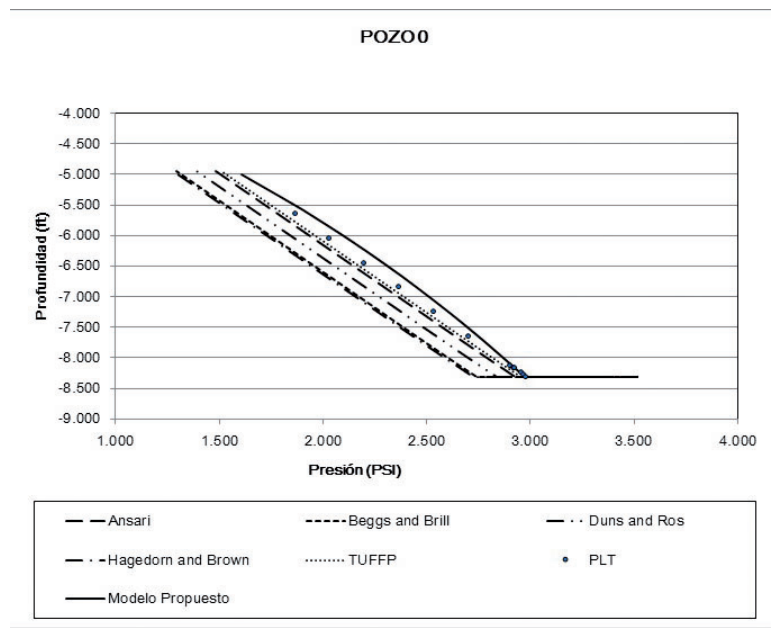

Figura 8. Resultados evaluación Pozo 0

Fuente: Autor

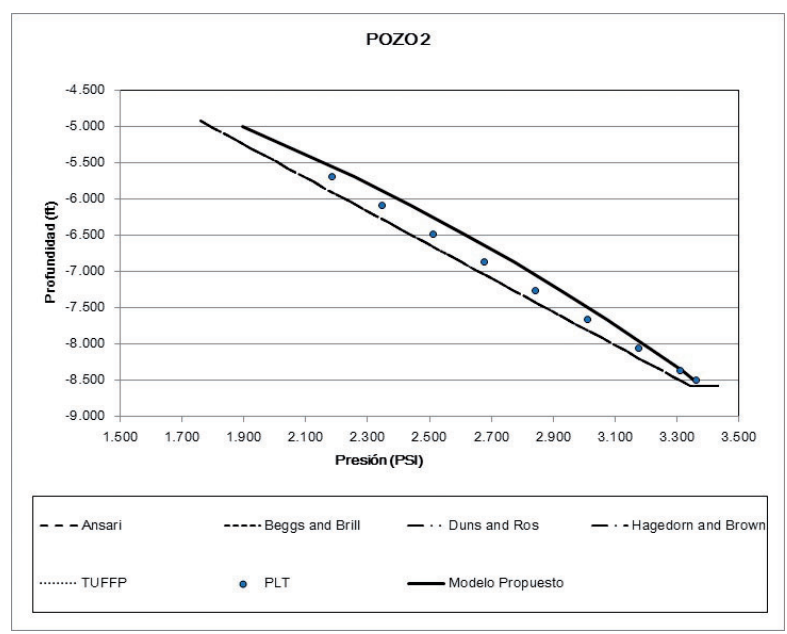

Figura 9. Resultados evaluación pozo 2

Fuente: Autor

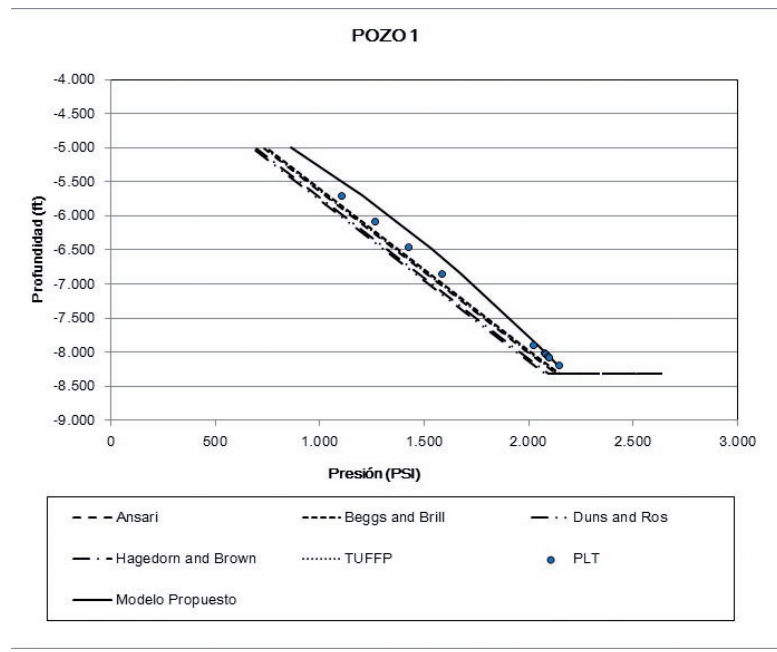

Figura 10. Resultados evaluación pozo 1 Fuente: Autor

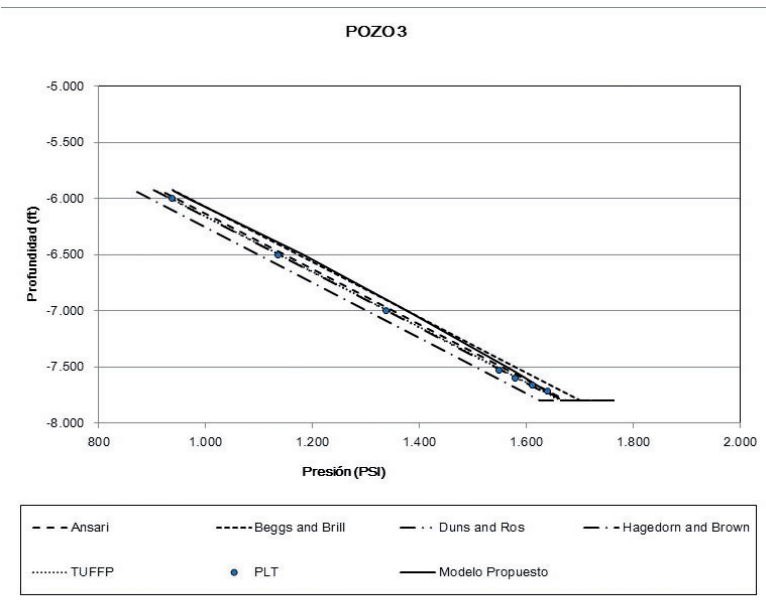

Figura 11. Resultados evaluación pozo 3

Fuente: Autor

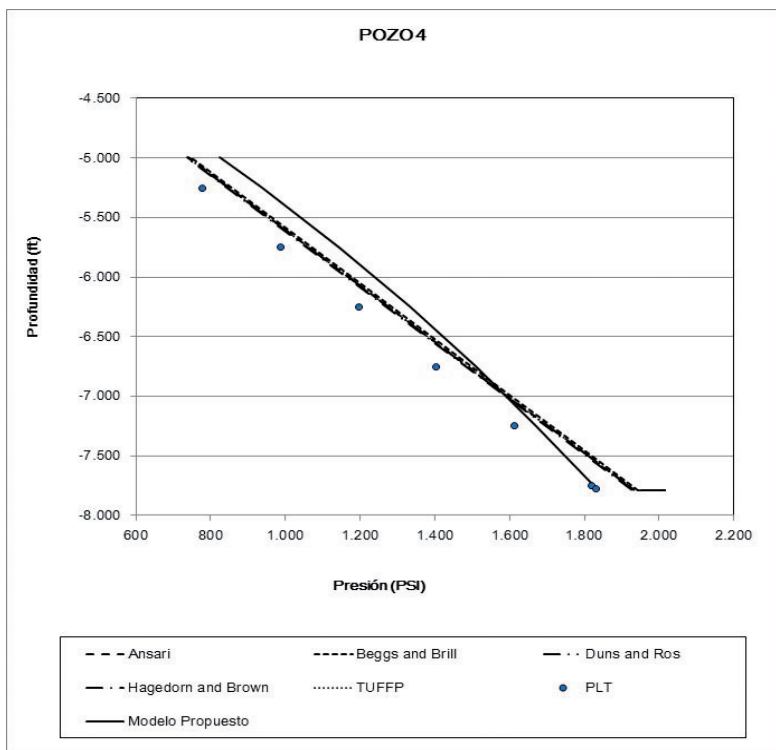

Figura 12. Resultados evaluación pozo 4 Fuente: Autor

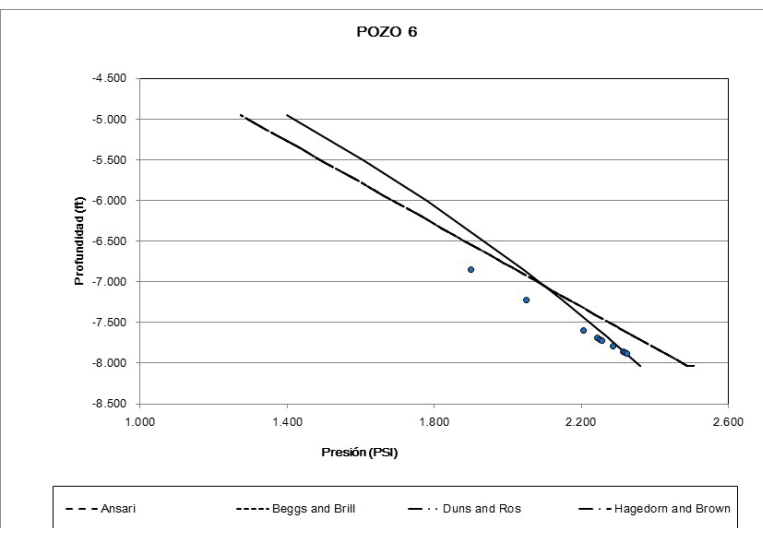

Figura 13. Resultados evaluación pozo 6

Fuente: Autor 


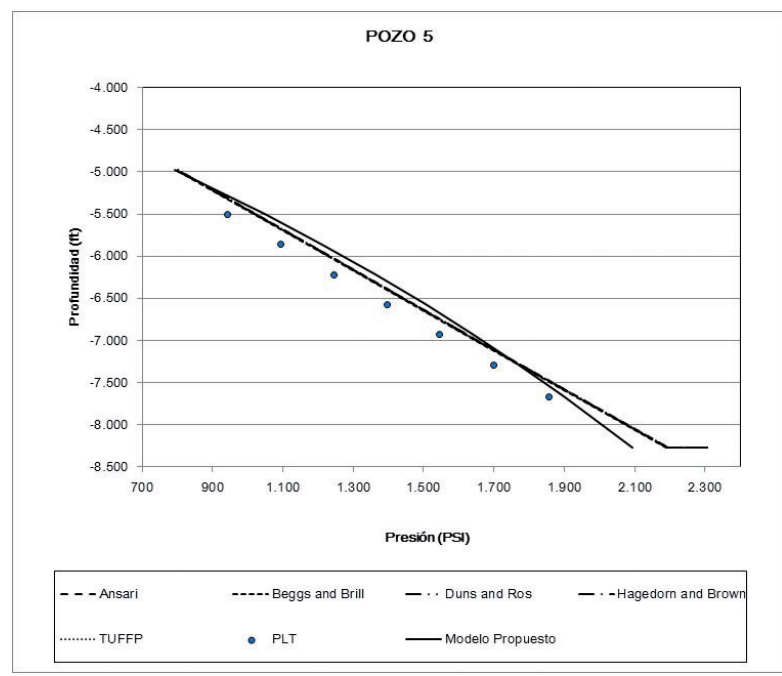

Figura 14. Resultados evaluación pozo 5

Fuente: Autor

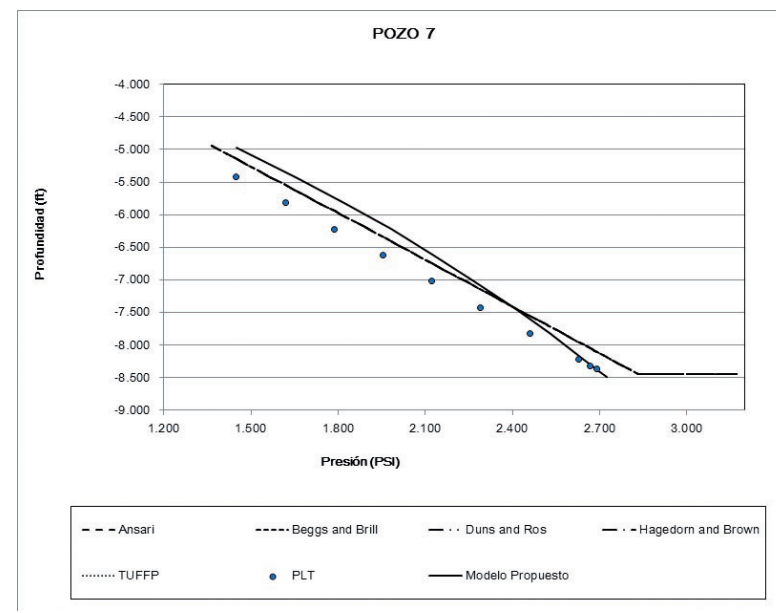

Figura 15. Resultados evaluación pozo 7

Fuente: Autor

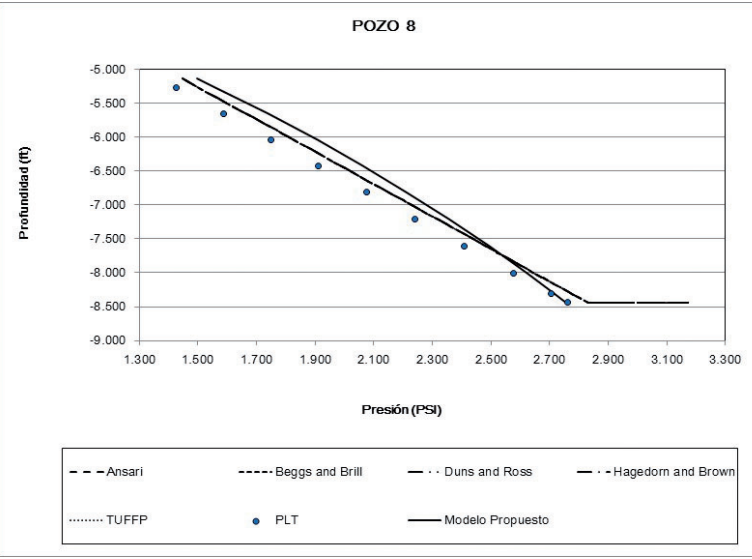

Figura 16. Resultados evaluación pozo 8

Fuente: Autor

\section{ANÁLISIS ESTADÍSTICO DE RESULTADOS}

La desviación de los valores comparados para los distintos pozos se muestra a partir del análisis del error utilizando los siguientes parámetros estadísticos:

\section{- Error relativo promedio (Er)w}

Este parámetro mide la desviación relativa respecto los datos experimentales:

$$
\boldsymbol{E} \boldsymbol{r}=\frac{1}{n} \sum_{i=1}^{n}\left(\frac{p_{\text {experimental }}-p_{\text {calculadd }}}{p_{\text {experimental }}}\right) * 100
$$

\section{- Error Relativo Absoluto (Ea)}

Este parámetro considera el valor absoluto de la desviación relativa respecto los datos experimentales:

$$
\boldsymbol{E} \boldsymbol{a}=\frac{1}{n} \sum_{i=1}^{n}\left|\frac{p_{\text {experimental }}-p_{\text {calculad } a_{i}}}{p_{\text {experimental }}}\right| * 100
$$

\section{- Desviación Estándar (DE)}

Es una medida de la dispersión de los datos calculados respecto al valor experimental:

$$
\boldsymbol{D E}=\sqrt{\frac{1}{n-1} \sum_{i=1}^{n}\left(\frac{\left.p_{\text {experimental }_{i}-p_{\text {calculad }_{i}}}\right)^{2}}{\text { experimental }_{i}}\right.}
$$

\section{- Coeficiente de determinación (R2)}

Es un parámetro simple que muestra el grado de ajuste de los datos calculados y experimentales en cuanto se aproxima el valor a 1 .

$$
\boldsymbol{R}^{2}=1-\frac{\sum_{i=1}^{n}\left(p_{\text {experimental }_{i}-} \text { calculadd }_{i}\right)^{2}}{\sum_{i=1}^{n}\left(p_{\text {calculada }_{i}}-\bar{p}\right)^{2}}
$$

Donde $\bar{p}$ es el valor de la media de los datos experimentales.

Los valores de estos parámetros nos permiten definir el comportamiento de los modelos evaluados: 
Tabla 4. Criterios de evaluación de desempeño de correlaciones para el modelamiento de la presión.

\begin{tabular}{ccccc}
\hline CORRELACIÓN & $\boldsymbol{E} \boldsymbol{E}$ & $\boldsymbol{E} \boldsymbol{a}$ & $\boldsymbol{D E}$ & $\boldsymbol{R}^{\mathbf{2}}$ \\
\hline ANSARI & -2.907 & 5.420 & 0.05978 & 0.94814 \\
\hline BEGGS AND BRILL & -2.392 & 6.411 & 0.07088 & 0.99979 \\
\hline DUNS AND ROS & -1.953 & 6.131 & 0.06798 & 0.94002 \\
\hline HAGEDORN AND BROWN & -1.171 & 6.809 & 0.07559 & 0.99977 \\
\hline TUFFP & -2.993 & 5.147 & 0.05668 & 0.94989 \\
\hline MODELO PROPUESTO & -5.273 & 5.410 & 0.06986 & 0.99985 \\
\hline
\end{tabular}

La variación del modelo propuesto se relaciona con las condiciones de operación de los pozos de la siguiente forma:

Respecto al GOR la variación del error relativo tiene una mayor densidad en los valores negativos, lo cual define que debido a los valores de GOR que se presentan se da una sobreestimación de los valores de presión con el modelo propuesto, de acuerdo a los valores registrados del PLT.

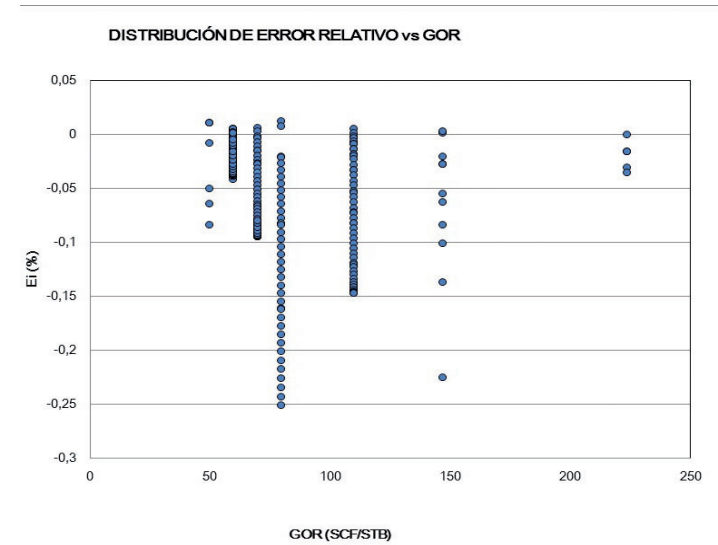

Figura 17. Distribución del error relativo en función del GOR Fuente: Autor

Esta misma tendencia se ve en la distribución de error en función de la densidad del líquido, como se ve en la siguiente figura:

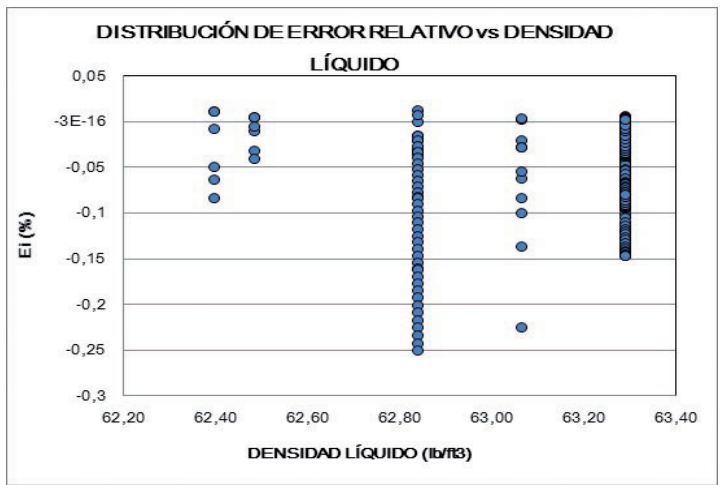

Figura 18. Distribución del error relativo en función de la densidad del líquido

Fuente: Autor
La sobreestimación de los valores de presión por el modelo propuesto se ve con mayor claridad en el diagrama que se muestra a continuación:

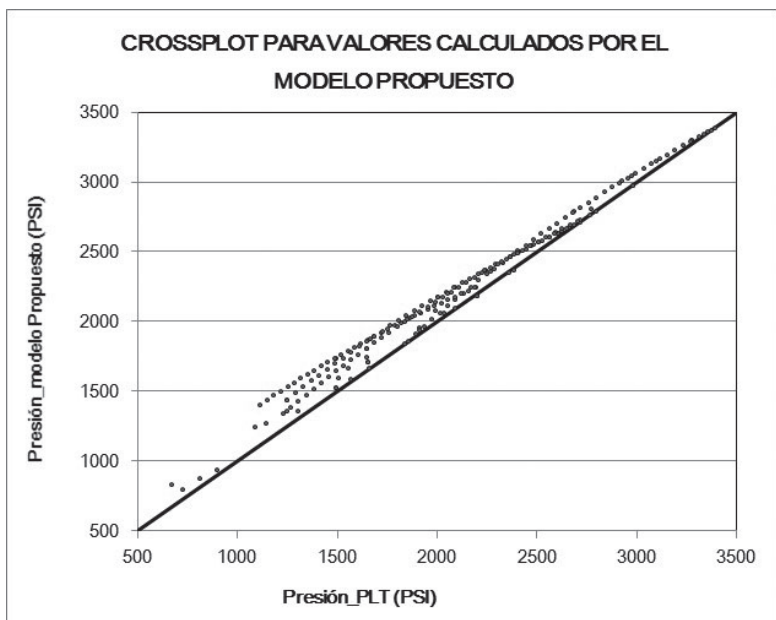

Figura 19. Crossplot para valores de presión calculados por el modelo propuesto

Fuente: Autor

La distribución de valores corresponde a un valor promedio de $6.2 \%$, lo cual define que el crudo de la formación productora de crudo extrapesado, no soporta la cantidad de gas que define el modelo propuesto, por lo cual, el valor de la presión cae más que el valor propuesto.

El análisis de sensibilidad del modelo propuesto, con base en los datos de los pozos evaluados, permite definir los siguientes rangos de implementación:

Tabla 5. Rangos de implementación de variables en el modelo.

\begin{tabular}{|lcc|}
\multicolumn{1}{c}{ Propiedad } & Valor & Unidad \\
\hline Fracción molar de gas & $0.1-0.7$ & \\
\hline Densidad de fluido & $8.5-10$ & ${ }^{\circ} \mathrm{API}$ \\
\hline Diámetros de tubería & $3.5-95 / 8$ & in \\
\hline BSW & $0-10$ & $\%$ \\
\hline Índice de comportamiento de flujo & $<1$ & \\
\hline Velocidad de deslizamiento & 0 & $\mathrm{ft} / \mathrm{s}$ \\
\hline Velocidad de deformación & $<500$ & $\mathrm{~s}-1$ \\
\hline
\end{tabular}


Los rangos de aplicación establecen que el modelo deberá reevaluarse para la definición del gradiente de presión que se produce al nivel de la bomba (Kraus, et al). El efecto sobre la reología debido a las altas velocidades de deformación producidas y el cambio de geometría del sistema, demanda un análisis numérico de una unidad de análisis diferente a la propuesta para esta investigación.

\section{CONCLUSIONES}

- El análisis de la reología de fluidos permitió establecer que los fluidos tipo Foamy Oil se comportan como no-newtonianos y puede usarse la ley de potencia, utilizados para caracterizar los fluidos tipo pseudoelásticos, modificando el índice de comportamiento de flujo ( en función del contenido de gas. La solución a la situación que se presenta cuando la velocidad de deformación () tiende a 0 , ocasionando que el valor de la viscosidad aparente tienda a infinito, lo cual es físicamente imposible, se consigue al asumir un valor límite inferior de contenido de gas. Este modelo funciona para contenidos de gas menores a $74 \%$, en donde el comportamiento es de tipo pseudoplástico respecto a la velocidad del líquido, y para contenidos de gas muy bajos el comportamiento es de tipo pseudoplástico respecto a la velocidad del gas.

- $\quad$ El fenómeno de nucleación se analizó a partir del balance de fuerzas que actúan sobre una burbuja de gas de geometría esférica en un medio líquido. El balance define la velocidad de ascenso de las burbujas de gas y mediante la velocidad relativa respecto a la velocidad del líquido se calculó la separación de las burbujas de un diámetro de burbujas de gas determinado. La derivación de la expresión de esta velocidad se dio a partir del desarrollo de la segunda ley del movimiento de Newton en donde la nucleación se presenta cuando la burbuja se acelera y se asume que se separa cuando la parte superior ha cubierto una distancia igual que el radio de las fuerzas balanceadas de la burbuja.

- El diámetro de separación se define mediante una función de distribución de probabilidad cuyo límite superior es el diámetro de la burbuja de Taylor, el cual corresponde a un patrón de flujo tipo slug (Brennen Christopher,). Este diámetro corresponde a un balance sobre la burbuja en donde las fuerzas de ascenso predominan en la dirección de flujo, con lo cual se obtiene una solución del valor del diámetro de esta burbuja a partir de una función cúbica (Mao, Z.S., and Duckler, A.E.,)..

- Se definió una función para la distribución del tamaño y la cantidad de las burbujas de tipo Weibul, en la cual el parámetro de forma () está en función de los diámetros de burbuja cuyo balance permite establecer condiciones de equilibrio dentro de la fase líquida y el parámetro de escala de la distribución () corresponde al diámetro de la burbuja de Taylor. Esta función actúa como factor de corrección de la modificación de la solubilidad de gas.

- El ajuste de las propiedades PVT del fluido tuvo en cuenta la definición de una pseudopresión de burbuja para describir la condición particular del fluido de mantener el gas en solución. Este valor se obtuvo a partir de la modificación de las constantes de equilibrio, siguiendo a Kraus et al., utilizando el software Hysys para encontrar, por medio de prueba y error, los valores de los parámetros Kvn.

- La ecuación de flujo en tubería utilizada para el desarrollo de esta investigación se derivó a partir de un balance sobre una unidad de análisis, en donde la relación entre las fases se define a partir de la Relación de Expansión Volumétrica () (Princen, H. M) . Este concepto permite incluir la función de distribución de tamaño y cantidad de burbujas de gas obtenida en esta investigación.

- La comparación del gradiente de presión determinado mediante la ecuación de flujo propuesta y otras correlaciones frente a datos experimentales de gradientes dinámicos tomados en una muestra de 9 pozos del campo productor de crudo extrapesado utilizado para la evaluación, muestra que la presión obtenida mediante la implementación del modelo propuesto tiene un valor medio de sobreestimación del $6.2 \%$.

- El análisis estadístico realizado corresponde a la sección de la tubería comprendida entre la cara de la formación productora de crudo extrapesado y la profundidad del intake de la bomba. La sobreestimación de los valores permite aseverar que este crudo tiene un comportamiento de tipo convencional, y el gas que permanece en solución a condiciones de cabeza de pozo puede analizarse atendiendo la precipitación de asfáltenos en este punto, los cuales actuarían como un surfactante. 


\section{REFERENCIAS}

1. Abou-el-hassan, M.E. Correlations for bubble rise velocity correlation, Chemical Engineer Communications, Vol. 2, Pág. 243, 1983.

2. Akagawa, K., and Sakaguchi, T., "Fluctuation in Void Ratio in Two-Phase Flow", Bulletin, JSME, Vol. 9, p. 104-120, 1966.

3. ANSYS ${ }^{\circledR}$ CFX-Solver. Release 10.0: Theory, 2010.

4. Barnes, H.A., Hutton, J.F. y Walters K., An Introduction to Rheology, Elsevier, 1989.

5. Bird, R.B. and Stewart, W.E. and Lightfoot, E.N.: "Transport Phenomena", John Wiley and Sons, Inc, NYC, 1960.

6. Blauer, R.E. and Holcomb, D.L.: "Foam Fracturing Shows Success in Gas, Oil Formations," Oil and GasJ. (Aug. 1975) 57-60.

7. Blauer, R.E., Mitchell, B.J. and Kohlhaas, C.A.: "Determination of Laminar, Turbulent,and Transitional Foam Flow Losses in Pipes", SPE 4885 presented at the 44th Annual California Regional Meeting of the Society of Petroleum Engineers of AIME, San Francisco, California, April 4-5, 1974.

8. Bonilla, L.F. and Shah, S.N.: "Experimental Investigation on the Rheology of Foams", SPE 59752 presented at the SPE/CERI Gas Technology Symposium held in Calgary, Alberta Canada, April 3-5, 2000 .

9. Brennen Christopher, Fundamentals of Multiphase Flows, California Institute of Technology, Cambridge University Press, 2005.

10. Calvert, J. R. and K. Nezhati, "A Rheological model for a liquid-gas foam," Int. J. Heat Fluid Flow 7, 164-168 (1986).
11. David, A. and Marsden, S.S.: "The Rheology of Foam", SPE 2544 presented at the 44th Annual Meeting of the SPE, Denver, CO, September 28, 1969.

12. Gaddis E.; Vogelpohl A., Bubble formation in quiescent liquids under constant flow conditions, Chemical Engineer Science, Vol 41, Págs. 97 $105 ; 1986$.

13. Gardiner, B.S., Dlugogorski, B.Z. and Jameson, G.J.: "Prediction of Pressure Losses in Pipe Flow of Aqueous Foams", Industrial and Engineering Chemistry Research, 38, p. 1099-1106, May 1999.

14. Harris, P.C. and Heath, S.J.: "High-Quality Foam Fracturing Fluids", SPE 35600 presented at the SPE Gas Technology Symposium held in Calgary, Alberta, Canada, April 28 - May 1, 1996.

15. Heller, J. P. and M. S. Kuntamukkula, "Critical Review of the Foam Rheology Literature," Ind. Eng. Chem. Res. 26, 318-325 (1987).

16. Kraus, W.P., Mc Caffrey, W.J., Boyd G.W.; Pseudo-Bubble Point Model for Foamy Oils, Paper CIM 93-45 presentado en CIM 44th Annual Technical Conference of the Petroleum Society of CIM, Calgary, Alberta, Mayo 9-12, 1993.

17. Mao, Z.S., and Duckler, A.E., "The Motion of Bubbles in Vertical Tubes. A Numerical Simulation for the Shape and Rise Velocity of Taylor Bubbles in Stagnant and Flowing Liquid", Journal of Computational Physics, Vol. 91, pp. 132-160, 1990.

18. Princen, H. M.. Rheology of foams and highly concentrated emulsions: I. Elastic Properties and Yield Stress of a Cylindrical Model System", Journal of Colloid Interface Science, Elsevier B.V., Vol 91, Págs. 160-175, 1983.

Recepción: 9 de septiembre de 2016

Aceptación: 3 de noviembre de 2016 